\title{
Vibration diagnostic system for evaluation of state interconnected electrical motors mechanical parameters
}

\author{
Oleksandr M. Vasilevskyi*a ${ }^{*}$ Pavlo I. Kulakov ${ }^{\mathrm{a}}$, Igor A. Dudatiev ${ }^{\mathrm{a}}$, Volodymyr M. Didych ${ }^{\mathrm{b}}$ Andrzej \\ Kotyra $^{c}$, Batyrbek Suleimenov ${ }^{\mathrm{d}}$, Azat Assembay ${ }^{\mathrm{d}}$, Ainur Kozbekova ${ }^{\mathrm{e}}$ \\ ${ }^{a}$ Vinnytsia National Technical University, 95 Khmelnitsky Shose str., 21000 Vinnytsia, Ukraine; \\ ${ }^{b}$ Vinnytsia National Medical University named after M. I. Pirogov, 56 Pyrohova str., 21000 \\ Vinnytsia, Ukraine; ${ }^{c}$ Lublin University of Technology, Lublin, Poland; ${ }^{d}$ Kazakh National Research \\ Technical University after K. I. Satpaev, 22 Satbaev str., 050013 Almaty, Kazakhstan; ${ }^{\mathrm{e}}$ Institute of \\ Information and Computational Technologies, 125 Pushkin str., 050010 Almaty, Kazakhstan
}

\begin{abstract}
The paper presents the structural diagram and mathematical model of a vibration diagnostic system to measure angular velocities of two interconnected electric motors. The system is based on vibration signals and the control signals of the motor mechanical parameters. The measurement procedure of the rotor rotational speed is based on vibration signals during synchronization. The procedure presented allows simultaneous measurement and synchronization frequencies of rotation to diagnose of the motors' mechanical parts. The calculated reduced error of synchronizing frequencies of rotation of the rotors, which is $0.45 \%$ of the measurement range of frequencies of rotation from 0 to $80 \mathrm{~Hz}$.
\end{abstract}

Keywords: vibration diagnostic, electric motors, asynchronism

\section{INTRODUCTION}

Development and research of vibration diagnostics systems for a state evaluation of the mechanical parameters of interconnected electric motors (EM) allows receiving timely and accurate information about the rotation frequency, asynchronism of rotation. Information about the actual state of EM is one of the components of the strategy for improving the efficiency of industrial-technological systems and reducing costs for their review during the operation. The proper maintenance of the condition of mechanical equipment can reduce the average cost of electric motors work support approximately 1.5 times compared to the cost of carrying out for this purpose of planning and preventive maintenance and almost 2 times compared with the cost of maintenance after failure.

The continuous growth in performance requirements of modern industrial production along with a wide introduction of EM vibration diagnostics necessitates opened new ways of reducing maintenance costs. Nowadays, simultaneous control of both rotation asynchronism and vibration diagnostic of two interrelated EM practically is still unexplored. The known works devoted to rotor systems vibration diagnostics ${ }^{1,2}$, automatic control of EM rotational speed ${ }^{3}$ do not allow simultaneous measurement of the rotation frequency of several EM, sync and perform the vibration diagnostic of the technical state of EM mechanical parameters.

An integrated approach to simultaneously measuring of the rotation frequency, automatic control of rotations asynchronism of interconnected EM and vibration diagnostic of a technical condition of electric motors have not been applied yet. Therefore, development of a diagnostic system for evaluating the state of mechanical parameters of an electric motor, which is also a new method of measuring the rotational speed is an actual scientific problem. The solution of such a problem will enhance their control, precision synchronization of EM rotational speed. What is more, it will reduce the maintenance costs

* o.vasilevskyi@gmail.com

Photonics Applications in Astronomy, Communications, Industry, and High Energy Physics Experiments 2017, edited by Ryszard S. Romaniuk, Maciej Linczuk, Proc. of SPIE Vol. 10445, 104456C

(C) 2017 SPIE · CCC code: 0277-786X/17/\$18 - doi: 10.1117/12.2280993 
The aim of the research is to develop a synchronization system for two electric motors that operation principle would be based on vibration acceleration signals, receiving the experimental results and the calculation error of asynchronous electric motors rotation of the rotors.

\section{THE METHOD APPLIED}

Positive simulation results of the automatic control of EM rotation asynchronism ${ }^{3,4}$, method of measuring the EM rotation frequency using of EM vibration metric parameters and the methods of forming of vibration diagnostic features of EM technical state ${ }^{5-7}$ allow developing vibration diagnostic system intended for state estimation of electric motors.

The most complete information about EM provides a measurement system that consists of four measuring channels of vibration (which consist of biaxial accelerometer (A), amplifier, a bandpass filter, a scale converter (SC), ADC), setpoint frequency (SF) of the EM rotor rotation, frequency regulators (FR) and digital signal processor (DSP) which handles vibration signals, synchronize rotor rotation frequency of interconnected EM and forms diagnostic features of EM technical state, and serial interfaces through which automatic adjustment (synchronization) of rotation frequency performs (Fig. 1).

The sequence of measuring transformation of the proposed vibration diagnostic measuring system can be described as follows. At the command of (SF) through an amplifier, a voltage-frequency converter U/F, DSP and FR the EM are started and their rotation frequency is regulated. Then with the help of high biaxial accelerometers, A1 and A2 the mechanical vibrations of each EM are measured which are amplified by preamp of charge and information bandwidth is allocated using the strip filters. The further vibration signal is scaled according to the selected ADC with scale converters (SC). After that, the ADC converts the signal into digital codes that are processed in the signal processor. To calculate the EM rotational speed using concentrically arranged piezoelectric accelerometers, inter-correlation function (ICF) is defined between measured vibration parameters on the axes of abscissas and ordinates $(X(t)$ and $Y(\mathrm{t}+\tau))$. If the measured vibration parameters are subjected to correlation is formed ICF $R_{X Y}(\tau)$ which shows the degree of correlation between two vibration signal depending on their relative shift (time of delay) $t$ in time domain ${ }^{5}$. The time at which the maximum of ICF is reached will display the delay $t$, by which the EM rotation frequency is estimated. ICF, which is used to calculate the rotation frequency of EM in DSP for vibration signal has a form:

$$
R_{X Y}(\tau)=\sum_{v=1}^{n} S_{X_{v}} S_{Y_{v}} \cos \left(\Psi_{v}-v 2 \pi f_{0} \tau\right)
$$

where $S_{X}$, and $S_{Y}$ denote RMS value of the measured vibration parameters $X(t)$ and $Y(t) ; \Psi_{v}$ - the phase shift between the $v$-th harmonics of signals examined, $\pi / 2 ; v$ - number of harmonics; $f_{0}$ - quantization frequency of the signal; $\tau$ - time delay between VS $X(t)$ and $Y(t)$ to calculate.

\section{RESULTS}

The obtained vibration signals were presented in Fig. 2 and 3. The inter-correlation function determined for these signals was calculated according to (1) are plotted in Fig. 4. The maximum of ICF is equal to the time delay ${ }^{6} t$. This time is calculated by DSP using improper integrals of normalized ICF $R_{X Y}(\tau)$ :

$$
\tau=\frac{1}{n T} \int_{0}^{n T} \frac{R_{X Y}(\tau)}{S_{X} S_{Y}} d \tau
$$

Substituting the formed ICF from equation (1) in the formula (2) it is possible to obtain the experimental value of the time delay which for the main EM (EM1) (Fig. 4a) equals $\tau=3.497 \mathrm{~ms}$, and for auxiliary EM (EM2) $-\tau=3.481 \mathrm{~ms}$ (Fig. $4 \mathrm{~b})$. Taking into account the known angle of piezoelectric accelerometers $(\pi / 2)$, the angular velocity of EM is defined as:

$$
\omega_{0}=\frac{\pi}{2 \tau}
$$

Thus, substituting the calculated values of the time delay in equation (3) the following experimental data can be obtained: $\omega_{1}=\pi /(2 \cdot 0.003497) \approx 449 \mathrm{rad} / \mathrm{s}$, which corresponds to rotation frequency of main electric motor $71.5 \mathrm{~Hz}, \mathrm{a}$ $\omega_{1}=\pi /(2 \cdot 0.003481) \approx 451 \mathrm{rad} / \mathrm{s}-$ corresponds to the rotation frequency of support electric motor $71.8 \mathrm{~Hz}$. The 
asynchronism of rotation of EM rotors rotation does not exceed $2 \mathrm{rad} / \mathrm{s}$ (19 rpm), and the relative error of an asynchronism of rotation makes $\delta=\Delta \omega / \omega_{1}=2 / 449 \approx 0.0045 \approx 0.45 \%$.

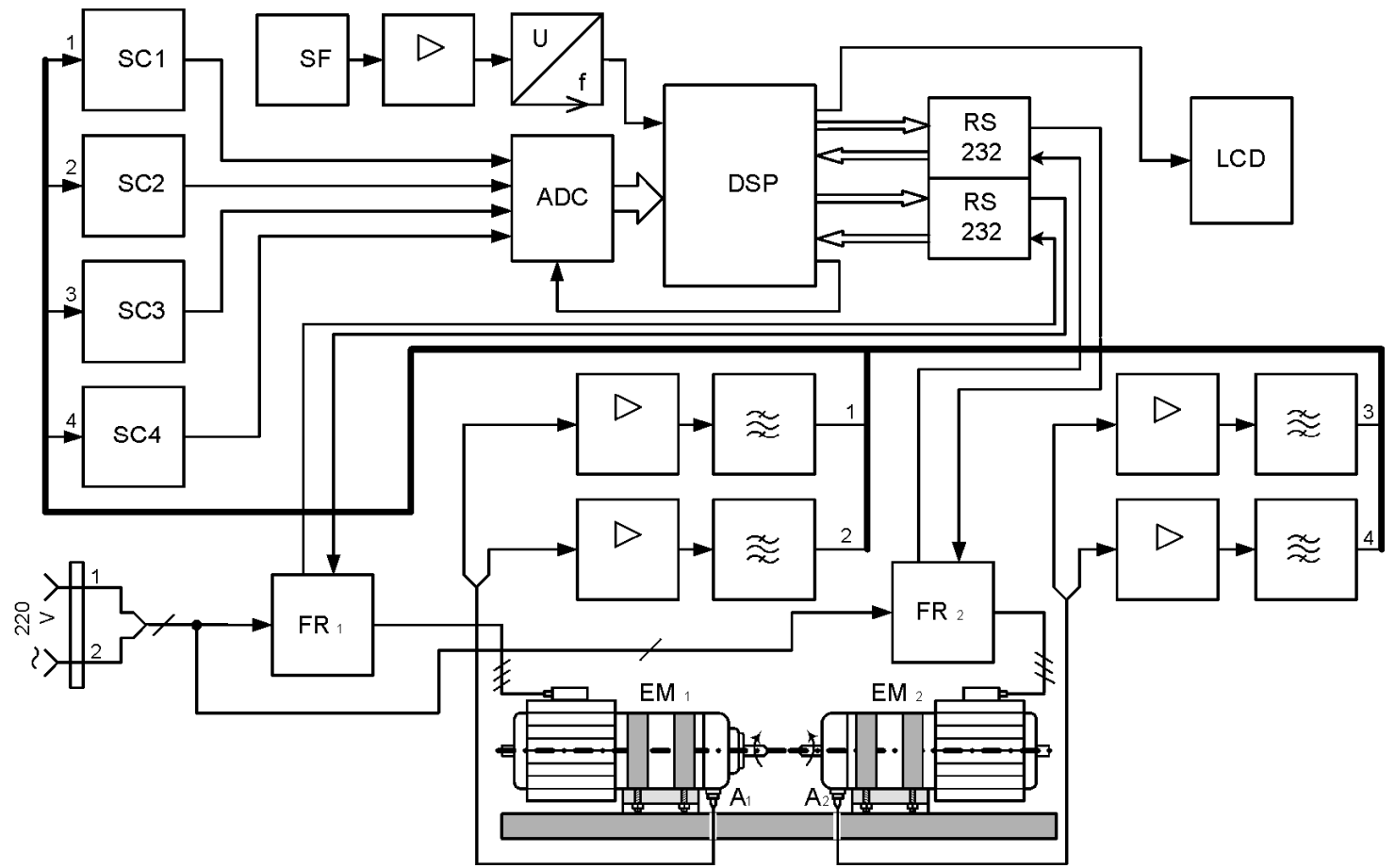

Figure 1. The structural diagram of vibration diagnostic system for evaluation of interconnected electric motors mechanical parameters.

The measured EM rotation frequencies are constantly compared to DSP and if the asynchronism of EM rotors rotation exceeds 20rpm then in the output of DSP digital code is formed to increase (or decrease) rotation frequency of auxiliary EM2 to that amount by which it rejected on the rotation frequency of main EM1. Then formed binary code transmitted via serial interface RS232 to control inputs of frequency regulator (FR2). After the appearance of the signal in FR2, it adapts to the rotation frequency of the main EM1 and thus, automatically controls the rotation of EM asynchronism. Thus, the difference of EM rotors rotation is automatically supervised. This cycle repeats always when measuring control system is working. When rotation difference exceeds 5 seconds, DSP generates an alarm signal on the LCD display (asynchronism of rotation exceeds the allowable limit). If it persists, it indicates that the EM is faulty. Faults of EM according to developed methods of the diagnostic features formation ${ }^{7,8}$ displayed on the LCD display.

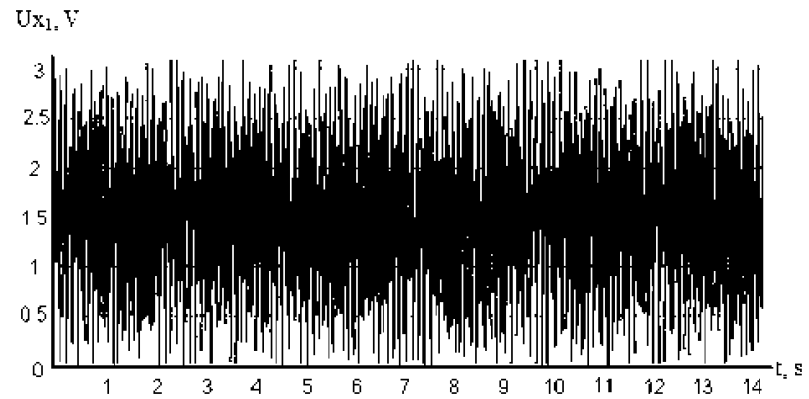

a)

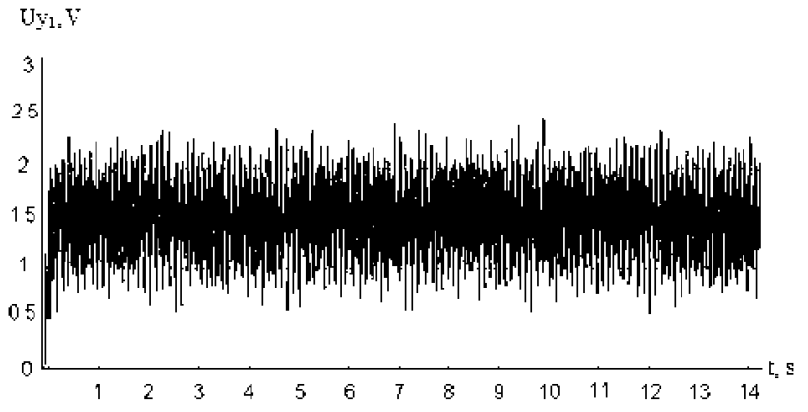

b)

Figure 2. The example vibration signals of the main electric motor (EM1): a) on the Y axis, b) on an X axis. 


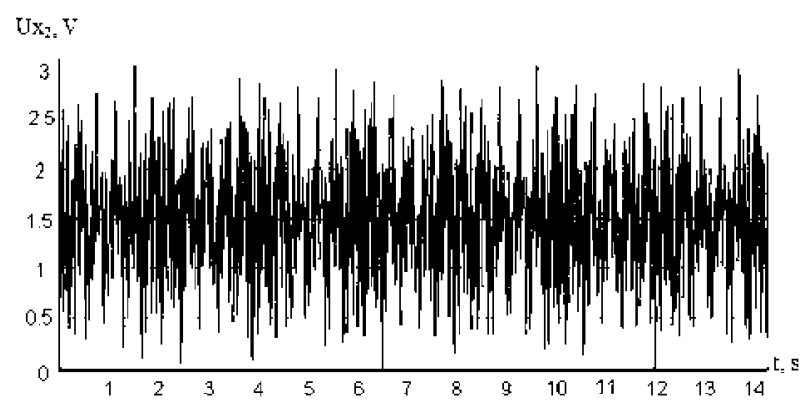

a)

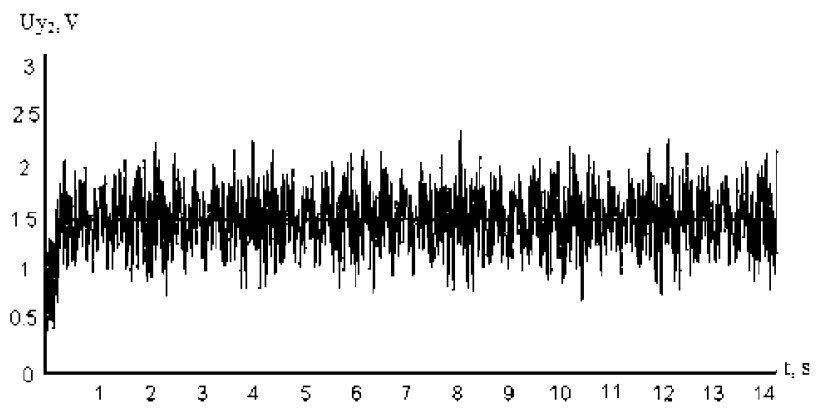

b)

Figure 3. The example vibration signals of the auxiliary electric motor (EM2): a) on the $\mathrm{Y}$ axis, b) on an $\mathrm{X}$ axis.

The results of three independent groups of experimental studies of EM rotation difference changing depending on given angular velocity are presented in Fig. 5. It can be noticed, the EM rotations asynchronism within the range of 0 to $80 \mathrm{~Hz}$ varies randomly. In the low-frequency range, it has lower values, whereas at the higher frequencies it tends to increase. Generally, it can be assumed that the difference of EM rotors rotation varies slightly.
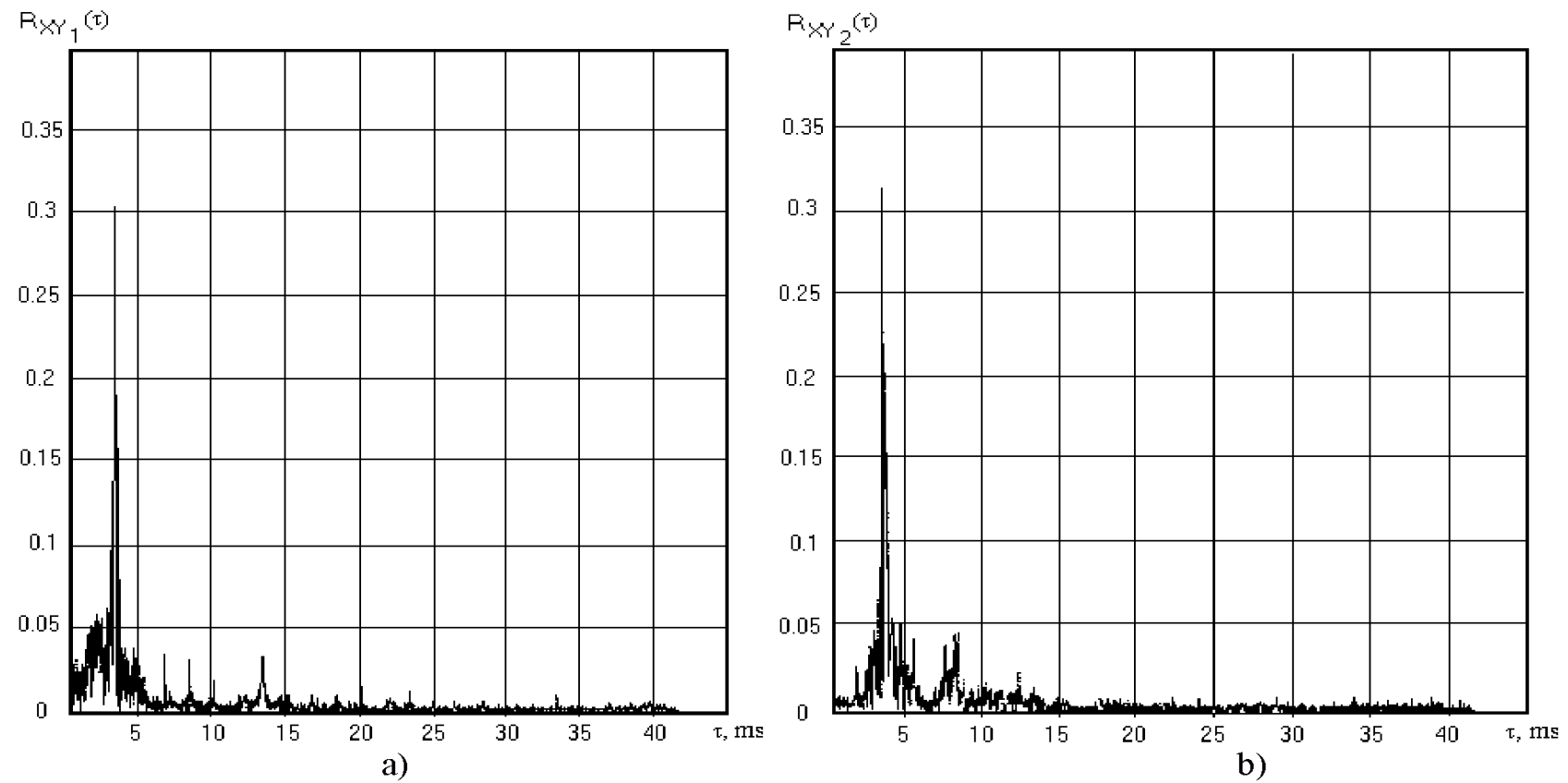

Figure 4. Experimental characteristics of the received inter-correlation function on the measured signals of vibration: a) main electric motor (EM1); b) auxiliary electric motor (EM2) on the frequency of rotation of $72 \mathrm{~Hz}$.

As it can be seen from experimental studies, the maximum absolute asynchronism of rotation does not exceed 20rpm $(\approx 2 \mathrm{rad} / \mathrm{s})$. Therefore, it can be assumed, the experimental error of a rotation difference in range 0 to $450 \mathrm{rad} / \mathrm{s}^{8}{ }^{8}$ :

$$
\gamma=\left|\Delta_{\max }\right| 100 \% / \omega_{\max }=|-2| 100 \% / 450=0.45 \%
$$

Thus, the analysis demonstrates that EM rotation asynchronism as the vibration signals is "periodically nonstationary," ie their characteristics change over rolling period, due to inertial properties of EM. Channels of vibration are identical to each other and work in the same conditions therefore in terms of studies of vibration channels properties, their characteristic can be considered as equal. 


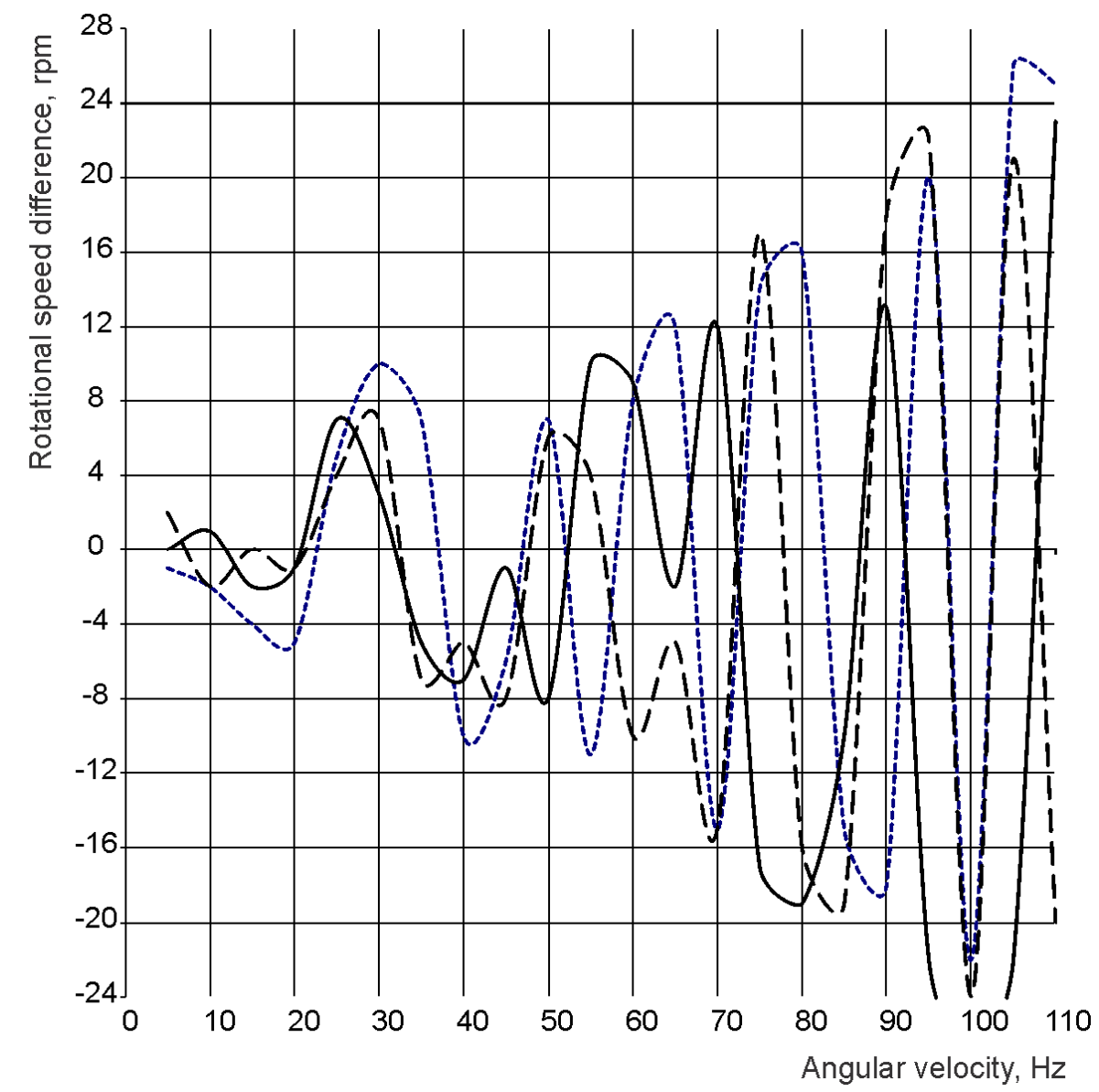

Figure 5. Experimental researches of change of a difference of rotation of curls of EM on different angular velocities.

\section{CONCLUSIONS}

The vibration diagnostic system was developed for a state estimation of mechanical parameters of interconnected electric motors. Its main element is a signal processor which allows to measure and handle vibration signals in real time. The developed vibration diagnostic system was used in experimental studies of vibration processes which occur in EM and was built experimental ICF based on which is calculated rotation frequencies as the main and auxiliary EM and is investigated asynchronism rotation at different frequencies.

As a result of the experiments, it was found that the resulted error of EM rotation frequency measurement after the vibration signals does not exceed $0.45 \%$. Such accuracy is possible only under the condition of precision accelerometers usage.

\section{REFERENCES}

[1] Stefanoiu, D. and Ionescu, F., "Mathematical Models of Defect Encoding Vibrations. A Tutorial" Journal of the American-Romanian Academy (ARA), 2001-2002 (2002).

[2] Barkov, A.V., Barkova, N.A. and Mitchell, J.S., "Condition Assessment and Life Prediction of Rolling Element Bearings," Sound \& Vibration 6, 10-17 (1995).

[3] Golnaraghi, F. and Kuo, B.C., [Automatic Control System], John Wiley and Sons Inc., Hoboken, 1-930 (2010).

[4] Vasilevskyi O.M., "Advanced mathematical model of measuring the starting torque motors," Tehnichna elektrodinamika 6, 76-81 (2013). 
[5] Podzharenko, V.O., Vasilevskyi, O.M. and Kucheruk V.Y., "Processing of vibration signals in digital measuring devices for determining the speed of electromechanical systems," Herald of Vinnitsia Polytechnic Institute 5, 5-9 (2004).

[6] Vasilevskyi, O.M., "Methodology to evaluate the durability of structures of power electric cars at random vibration load," Herald of Vinnitsia Polytechnic Institute 5, 58-61 (2010).

[7] Zagirnyak, M., Prus, V., Kolotylo, I. and Miljavec, D., "Taking stator cores properties into account when induction motors vibration parameters are calculated," Przeglad Elektrotechniczny 12, 192-195 (2013).

[8] Podzharenko, V.O. and Vasilevskyi, O.M., "Diagnostics of technical condition of electromechanical systems for the logarithmic decrement," Proceedings of Donetsk National Technical University; Series: Computers and Automation 88, 138-144 (2005). 